\title{
Novel neutron detector assembly based on SiPM readout to be coupled with the Active Target for SPES
}

\author{
Felix Pino ${ }^{1}$, Jessica Delgado ${ }^{1}$, Giorgia Mantovani ${ }^{1}$, Lucio Pancheri ${ }^{2}$, Daniela Fabris ${ }^{3}$, Cristiano L. \\ Fontana $^{4}$, Vladimir Ruiz ${ }^{1}$, Davide Brunelli ${ }^{2}$ and Sandra Moretto ${ }^{1,3}$ \\ ${ }^{1}$ Department of Physics and Astronomy "Galileo Galilei”, University of Padova, Italy \\ ${ }^{2}$ Department of Industrial Engineering, University of Trento, Italy \\ ${ }^{3}$ INFN Sezione Padova, Italy \\ ${ }^{4}$ European Commission, Joint Research Centre, Geel, Belgium \\ felixeduardo.pinoandrades@unipd.it
}

\begin{abstract}
The Active Target ATS (Active Target for SPES) is a new time-projection chamber designed for reaction and decay studies with nuclei far from stability. The physics cases for the new-generation active target are related to the ongoing developments of facilities for radioactive ion beams. Thanks to its flexibility, this instrument will be capable of taking advantage of the most exotic beams which will become available at the SPES facility under construction at the Legnaro National Laboratories in Italy. Particular attention will be also paid to couple it with ancillary detectors, for both charged and neutral (gamma and neutrons) particles. In particular, in this work, we will focus the attention on the neutron ancillary detectors. The proposed prototype is a compact device able to discriminate, by performing pulse shape analysis, between neutrons and gamma. The device take advantage of recent improvements in silicon photomultiplier (SiPM) technology and the development of new plastic scintillators exhibiting neutron/gamma discrimination capability. Our work is focused on the read-out with silicon photomultipliers arrays of EJ-276 (and its old version EJ-299) and EJ-276G scintillators of several sizes (ranging from $20 \mathrm{~mm}$ to $50 \mathrm{~mm}$ diameter). Moreover, we will show the comparison of discrimination performances between SiPM and standard photomultiplier read-out configurations.
\end{abstract}

Keywords —Neutron/gamma discrimination, SiPM, plastic scintillators, neutron detector.

\section{INTRODUCTION}

$\mathrm{T}$ HE SPES (Selective Production of Exotic Species) project is conceived as a multidisciplinary facility that is being installed at Legnaro National Laboratories (INFN, Italy) [1]. It has been designed as a second-generation Radioactive Ion Beam (RIB) facility using the Isotope Separation On-Line (ISOL) technique. A thick target (Uranium Carbide) is bombarded with a high intensity $(\sim 250 \mu \mathrm{A})$ primary proton beam $\left(E_{p} \sim 30-70 \mathrm{MeV}\right)$ to induce up to $10^{13}$ fissions/s. The project is organized in four phases: $\alpha, \beta, \gamma$ and $\delta$. SPES- $\alpha$ spans the installation of the proton driver (cyclotron produced by the Best Cyclotron Systems Inc.). SPES- $\beta$ covers the production and post-acceleration of RIBs. SPES- $\gamma$ includes the production of radionuclides focused mainly for medical applications. And, SPES $-\delta$ covers the production of neutron fields for applied science studies (material science, biology, etc.).

In the framework of the SPES- $\beta$ phase, which is focused on the use of high intensity and high-quality neutron-rich nuclei beams to perform forefront research in nuclear structure and reaction dynamics, there is an ongoing project aimed to design and build an ACtive TARget and Time Projection Chamber (ACTAR TPC) [2,3]. This device is a novel gas-filled detector, and at the same time a gaseous thick target, that allows the tracking of charged particles in three dimensions and provides a precise reaction energy reconstruction from the vertex position. For many cases of interest [4, 5], coupling ancillary detectors (for neutrons and gamma) to the ACTAR TPC can help to optimize the data acquisition and to improve the precision of the results. In particular, in the present work, we will focus the attention on the neutron ancillary detectors. We propose a compact detector able to discriminate, by performing pulse shape analysis, between fast neutrons and gamma photons. The device take advantage of recent improvements in silicon photomultiplier (SiPM) technology and the development of new plastic scintillators exhibiting neutron/gamma discrimination capability.

Organic scintillators with discrimination capabilities exhibit a fast decay component $\left(\tau_{f} \sim\right.$ tens of $\left.n s\right)$ and a slow decay component $\left(\tau_{s} \sim\right.$ hundreds of ns). The last one is the most important to discriminate particles, because its intensity changes as a function of the linear energy transfer $(\mathrm{dE} / \mathrm{dx})$ of the particle that excites the scintillator. The pulse shape discrimination technique is based on the reconstruction of the time profile of the scintillation decay. With fast photomultipliers (rise time $\sim$ units of ns) this can be done in a straightforward way, but when using SiPM there are several aspects to take into account: the number and recovery time of the microcells, and the dimension (active area) of the SiPM. In a SiPM the output signal is made of the sum of the individual signals coming from the fired microcells. Organic scintillators produce $\sim 10000$ photons/ $1 \mathrm{MeV} \mathrm{e}^{-}$, so, in order to collect most information we need at least the same order of magnitude of microcells. On the other hand, the recovery time of the microcells goes from 10 to $100 \mathrm{~ns}$ depending on the equivalent RC circuit. Shorter recovery time could potentially help the 
performance of the SiPM in terms of particle discrimination with organic scintillators. Collection of the total scintillation light is important to get accurate reconstruction of time profiles, so, using SiPM arrays is necessary in order to increase the active area up to units of $\mathrm{cm}^{2}$, and be able to read-out large-sized scintillators.

\section{EXPERIMENTAL SET-UP}

\section{A. Plastic Scintillators}

Three types of organic plastic scintillators with neutron/gamma discrimination capabilities, from Eljen Technology - Texas US, were used. The scintillators have cylindrical shapes and several dimensions, we used: two EJ-299 (20 mm dia. x $20 \mathrm{~mm}$ thick. and $50 \mathrm{~mm}$ x $50 \mathrm{~mm}$ ) acquired in 2013 and 2015 respectively, one EJ-276 (25 mm dia. x $25 \mathrm{~mm}$ thick.) and one EJ-276G (25 mm dia. x $25 \mathrm{~mm}$ thick.), these last two acquired in 2019. Note that the EJ-276 model replaced the EJ-299 one. The producer declared that the improvements were mostly in terms of physical hardness and long-term stability of scintillation and optical characteristics. Essentially, features like density, scintillation efficiency and emission curves are very similar.

\section{B. Silicon Photomultiplier Arrays}

Two types of silicon photomultipliers arrays, from AdvanSiD - Trento IT, were tested. A near ultra-violet (NUV) and a red-green-blue sensitive (RGB) SiPM. The main characteristics of the SiPM can be seen on Table 1. In Fig. 1 the photon detection efficiency (PDE) of the SiPM and the emission curves of the plastic scintillators are reported. As can be seen, there is an excellent match between the EJ-276 emission and the photon detection efficiency peak of the NUVSiPM (as happen with the standard photomultiplier). On the other hand, the EJ-276G emission is between the sensitivity peak of the NUV and RGB-SiPM. For comparison purposes, a 2" fast linear focused photomultiplier (PMT) Hamamatsu, model H1949-51, was used.

TABLE I

MAIN CHARACTERISTICS OF THE SILICON PHOTOMULTIPLIER ARRAYS USED

\begin{tabular}{ccc}
\hline & NUV-SiPM & RGB-SiPM \\
\hline Array size & $16.88 \mathrm{~mm} \times 17.43 \mathrm{~mm}$ \\
Single SiPM size & $4 \mathrm{~mm} \times 4 \mathrm{~mm}$ \\
Cell number & $9340 / \mathrm{ch}$ \\
Cell size & $40 \mu \mathrm{m} \mathrm{x} 40 \mu \mathrm{m}$ \\
Recharge time constant & $70 \mathrm{~ns}$ & $50 \mathrm{~ns}$ \\
Peak sensitivity wavelength & $420 \mathrm{~nm}(43 \%)$ & $550 \mathrm{~nm}(32.5 \%)$ \\
Breakdown voltage, typ & $26 \mathrm{~V}$ & $27 \mathrm{~V}$ \\
Dark count rate & $<100 \mathrm{kHz} / \mathrm{mm}^{2} @ \quad<200 \mathrm{kHz} / \mathrm{mm}^{2}$ \\
& $4 \mathrm{~V} \mathrm{OV}$ & @ 4V OV \\
\hline \hline
\end{tabular}

\section{Read-out board for the SiPM $4 \times 4$ arrays}

The read-out board for the SiPM arrays is divided in two parts: the connection of all 16 pixels of the SiPM array (to sum the single signals and to supply the bias voltage) and, the amplification of the anode signal, see Fig. 2. According to [6], connecting in parallel four sets made of 4 single SiPM connected in series is the optimal configuration to get a good sum signal, in terms of its amplitude and timing characteristics. Also, in this way the supply of the required bias voltage to each pixel can be managed easily. By the other hand, the other part of the read-out board consists in a transimpedance preamplifier based on a Ultra-Low Noise, High-Speed OpAmp (LMH6629). The circuit was simulated theoretically and tested illuminating the active area of the SiPM array with an attenuated pulsed-laser (width $\sim 4 \mathrm{~ns}$ ). An excellent agreement was found between the theoretical predictions and the experimental signals. These exhibit a fast leading edge $(<10 \mathrm{~ns})$ and a decay time dominated for the recovery time of the $\operatorname{SiPM}(\sim 100 \mathrm{~ns})$.

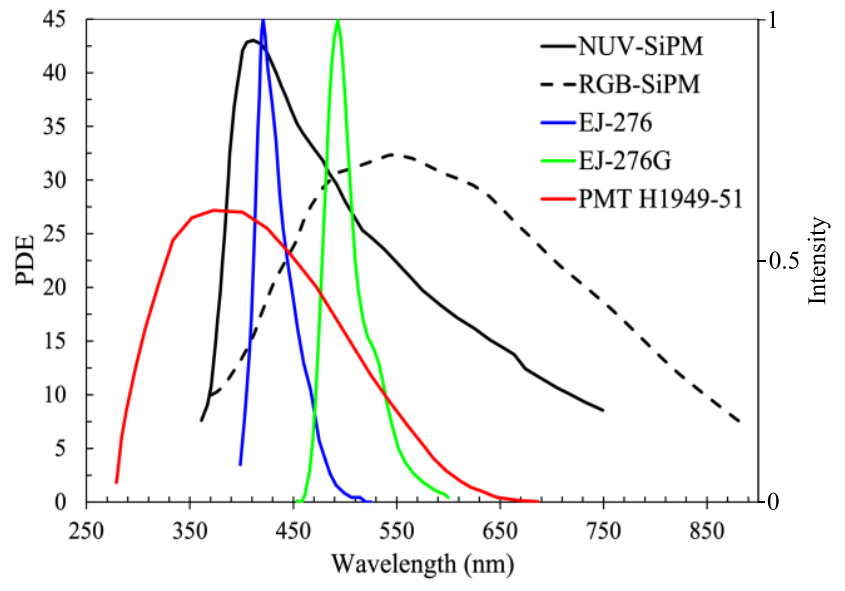

Fig. 1. Photon detection efficiency (PDE) corresponding to the NUV and RGB SiPM (left axis) compared with the emission curves of the plastic scintillators (right axis). Quantum efficiency of the H1949-51 PMT is also given (left axis).

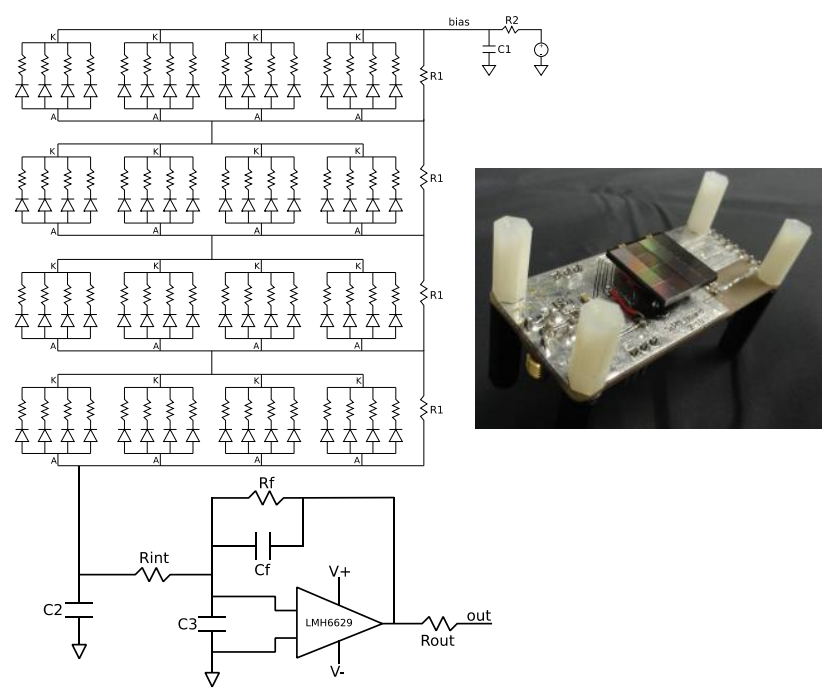

Fig. 2. Scheme (left) and photo (right) of the transimpedance preamplifier used to read-out the anode signals coming from the SiPM arrays. 


\section{DAQ and data processing}

Output signals (coming from the PMT or the transimpedance amplifier) were acquired using a CAEN DT5725 fast digitizer (250 MSamples/s, 14-bit ADC resolution). The digitizer allows to record the signals as digitized waveforms, to perform subsequent off-line analysis. Also, the installed firmware (Digital Pulse Processing for Charge Integration and Pulse Shape Discrimination, DPP-PSD) can pre-process the data, determining the pulses timestamps and integrating them over two gates, in order to perform particle discrimination using the so-called double integration method [7].

Bias voltage was applied using a CAEN V6533 VME HV Power Supply Module. A CAEN V1718 VME-USB 2.0 Bridge was used to control the HV power supply unit. A CAEN A3818 PCI Express CONET2 Controller installed on a PC was used to control the digitizer (by means of optical fiber connections using the CAEN CONET2 protocol). All parameters of the nuclear electronics, and also of the data acquisition, were managed using the ABCD (Acquisition and Broadcast of Collected Data) software [8-10], released as an open-source project (https://github.com/ec-jrc/abcd). The OpAmp was powered using the a power supply (Oltronix) model B703DT.

A ${ }^{22} \mathrm{Na}$ calibration gamma source $(\sim 300 \mathrm{kBq})$ and a $\mathrm{AmBe}$ source (neutron emission rate $\sim 2 \times 10^{5} \mathrm{~s}^{-1}$ ) were used for the measurements. In Fig. 3 a typical experimental set-up is presented. As can be seen, the plastic scintillator was wrapped with Teflon tape (PTFE) to improve the light collection. The assembly scintillator/SiPM/read-out board was located inside a black box to isolate it from external light (not shown in Fig. 3).

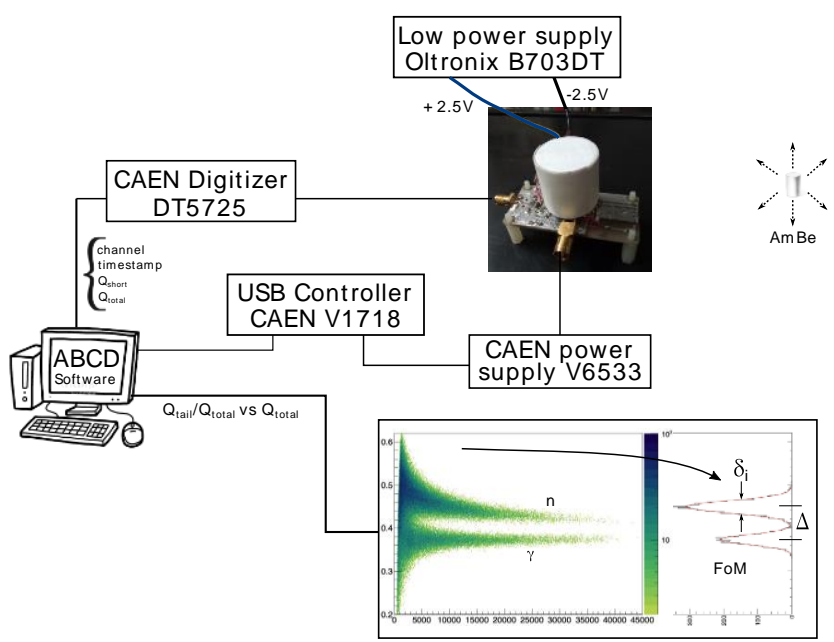

Fig. 3. Experimental set-up. 1" x 1" EJ-276 scintillator coupled to the NUV$\mathrm{SiPM}$ array is shown.

The double gate integration method was applied to perform the pulse shape discrimination analysis. The pulse shape parameter (PSP) is computed as: PSP $=Q_{\text {tail }} / Q_{\text {total }}$, where $Q_{\text {tail }}$ is the integral over the tail of the signal (computed as: $\left(Q_{\text {total }}\right.$ $\left.Q_{\text {short }}\right) . Q_{\text {short }}$ is the integral over a short gate, including the fast rise time region and part of the fastest decay component of the pulse, and $Q_{\text {total }}$ is the integral over a long gate, encompassing the majority of the pulse.
In order to characterize the discrimination capability of the scintillator/read-out device assembly, the Figure of Merit (FoM), defined as FoM $=\Delta /\left(\delta_{n}+\delta_{\gamma}\right)$, was computed by analyzing the PSP distribution of events with energies between $1 \mathrm{MeVee} \pm 20 \% . \Delta$ is the difference between the two mean values of the neutron and gamma distributions and $\left(\delta_{n}+\delta_{\gamma}\right)$ is the sum of the gamma and neutron Full Width at Half Maximum (FWHM).

\section{RESULTS AND ANALYSIS}

\section{A. EJ-299, $20 \mathrm{~mm}$ dia. $\times 20 \mathrm{~mm}$ thick.}

The first thing that we want to compare is the shape of the signals coming from the PMT and from the transimpedance preamplifier output (SiPM). For this purpose we used the smaller plastic scintillator. In Fig. 4 average neutron and gamma induced signals coming from the PMT and the NUVSiPM array can be seen. PMT signals decay is very fast, it is dominated by the fast decay component of the scintillator ( units of ns). By the other hand, the decay of the SiPM signals is much slower ( hundreds of ns), in this case it is dominated for the recovery time of the microcells of the SiPM, however, good differences between neutron and gamma induced signals can be observed.

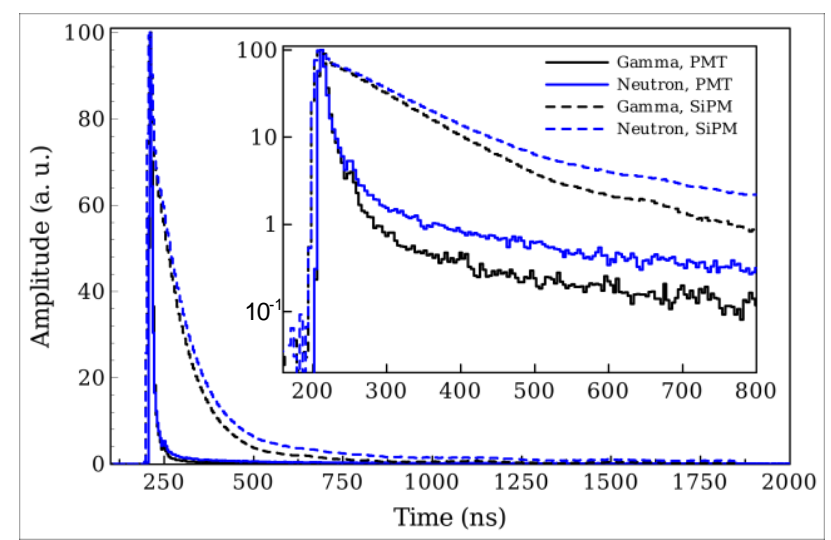

Fig. 4. Neutron and gamma average signals coming from the PMT anode and preamplifier output (NUV-SiPM) using an EJ-299 (20 mm dia. x 20 mm thick.).

In order to cover as much as possible the scintillator face, our first tests were conducted using a small plastic scintillator. In this specific case we were able to cover $\sim 87 \%$ of the area. In Fig. 5 the ${ }^{22} \mathrm{Na}$ energy spectra acquired with the small EJ-299 coupled to the PMT and to the NUV-SiPM array are shown. As can be seen the energy resolution obtained with the SiPM array is very good. Qualitatively it can be evaluated using the magnitude of the slope of the Compton edges, larger slopes mean better energy resolution. By following this approach, the small EJ-299 exhibits slightly better energy resolution when it is coupled to the NUV-SiPM array. This result is mainly due to the combination of covering a large fraction of the scintillator face and the high PDE of the SiPM (see Fig. 1).

To assess the neutron/gamma discrimination performance of 
the small EJ-299 coupled to the NUV-SiPM array we did some measurements using the Am-Be source (shielded with $5 \mathrm{~cm}$ of $\mathrm{Pb}$ to attenuate the $59.5 \mathrm{keV}$ gamma intensity). In Fig. $62 \mathrm{D}$ plots, PSP versus $Q_{\text {total }}$ (proportional to the light output), corresponding to the measurements when the scintillator was coupled to the PMT (a) and to the NUV-SiPM array (b) are reported. SiPM bias voltage was set in $120 \mathrm{~V}$, i.e. overvoltage $(\mathrm{OV})$ equal to $4 \mathrm{~V}$. As can be seen excellent $\mathrm{n} / \gamma$ discrimination is found in both cases.

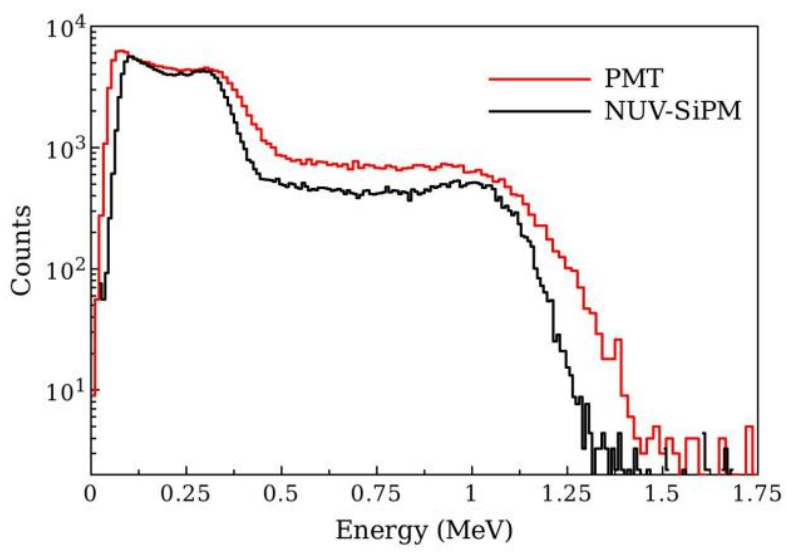

Fig. 5. ${ }^{22} \mathrm{Na}$ spectra using the PMT and the NUV-SiPM array coupled to the EJ299 (20 mm dia. x 20 mm thick.).
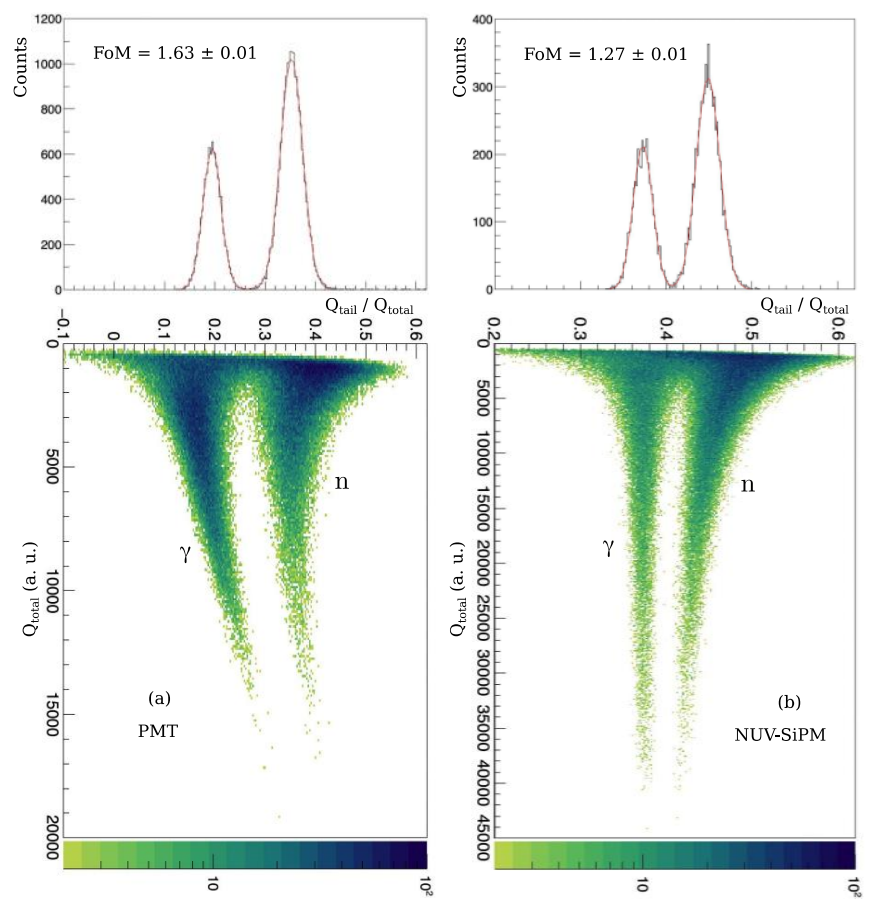

Fig. 6. 2 D plots, PSP versus $Q_{\text {total }}$, corresponding to the measurements using the Am-Be source with the EJ-299 (20 mm dia. x $20 \mathrm{~mm}$ thick.) coupled to the PMT (a) and to the NUV-SiPM array (b). PSP distributions and FoM values (at $\sim 1 \mathrm{MeVee}$ ) are reported on top.

The short and long integration gates in the case of the NUVSiPM were optimized and found to be $100 \mathrm{~ns}$ and $720 \mathrm{~ns}$ (measured from the leading edge of the signal) respectively. FoM value (at $\sim 1 \mathrm{MeVee}$ ) obtained with the NUV-SiPM array is $\sim 80 \%$ respect to the one we got when using the PMT. The results exceed the performance reported by [11], using a SensL SiPM array, and are consistent with [12], using a cubic $(6 \mathrm{~mm}$ x $6 \mathrm{~mm}$ × $6 \mathrm{~mm}$ ) plastic scintillators and SensL C- and J-series SiPM arrays, and [13], using Hamamatsu SiPM arrays.

\section{B. EJ-276 and EJ-276G, $25 \mathrm{~mm}$ dia. $x 25 \mathrm{~mm}$ thick.}

Our next step was to study the $\mathrm{n} / \gamma$ discrimination capability of the assembly composed of a $25 \mathrm{~mm}$ dia x $25 \mathrm{~mm}$ thick. EJ276 plastic scintillator coupled to the NUV-SiPM array. In this case the covered area of the scintillator face is $\sim 60 \%$, even so, similar results, in terms of energy resolution and $n / \gamma$ discrimination, were obtained compared to the smaller EJ-299 scintillator. In Fig. 7 the $2 \mathrm{D}$ plots, PSP versus $Q_{\text {total }}$, corresponding to the measurements (with the Am-Be source) when the scintillator was coupled to the PMT (a) and to the NUV-SiPM array (b) are given. Here a good FoM value $(\sim 1$ at $\sim 1 \mathrm{MeVee}$ ) was found using the NUV-SiPM, that is $\sim 80 \%$ the one obtained with the standard PMT. This means that the lack of light collection was compensated by the high PDE of the SiPM, letting a good separation between neutrons and gamma events. These results are consistent with data reported using a larger SiPM (8x8 array from SensL), in which slightly better results have been obtained [14].
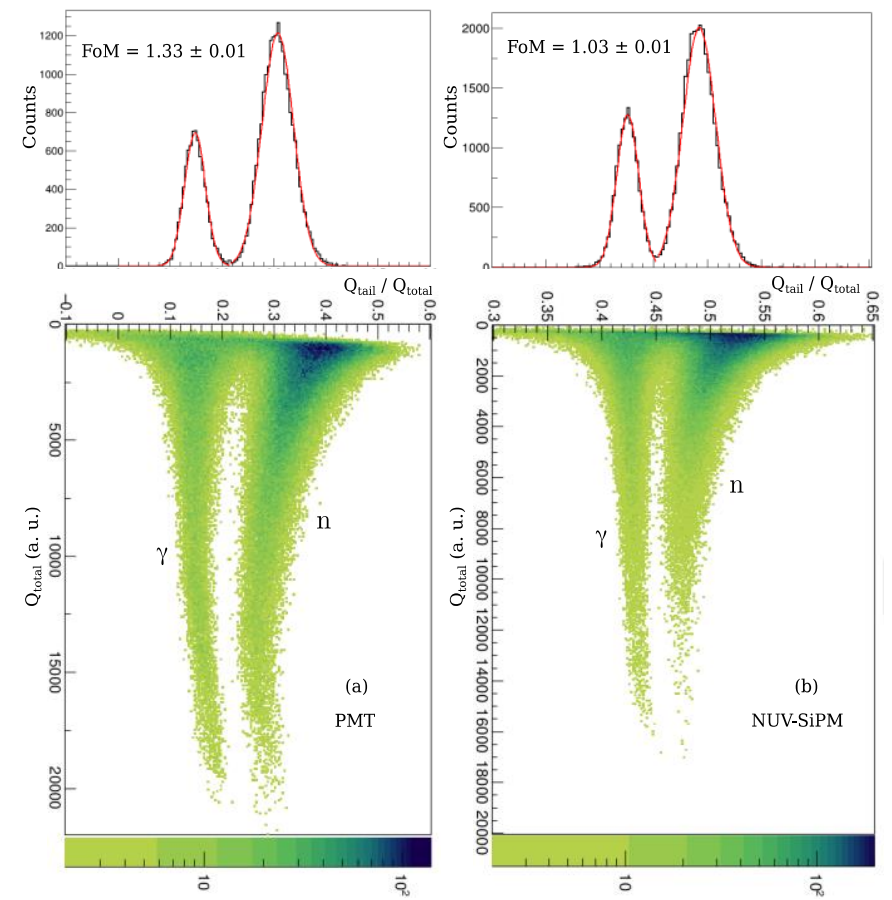

Fig. 7. 2D plots, PSP versus $Q_{\text {total }}$, corresponding to the measurements using the Am-Be source with the EJ-276 (25 mm dia. x $25 \mathrm{~mm}$ thick.) coupled to the PMT (a) and to the NUV-SiPM array (b). PSP distributions and FoM values (at $\sim 1 \mathrm{MeVee}$ ) are reported on top.

Another interesting study was carried out with the EJ-276G scintillator (25 mm dia. x $25 \mathrm{~mm}$ thick.). It is known as the green emitting EJ-276 scintillator, however, as can be seen in Fig. 1 its emission is balanced between blue and green 
wavelengths. For this reason, NUV and RGB types of SiPM arrays were tested in order to find the optimum assembly to get better $\mathrm{n} / \gamma$ discrimination.

In this case, when the scintillator was read-out with the PMT the FoM value (at $\sim 1 \mathrm{MeVee}$ ) was $(1.16 \pm 0.01)$. In Fig. 8 the 2D plots, PSP versus $Q_{\text {total }}$, corresponding to the measurements (with the Am-Be source) when the scintillator was coupled to the NUV-SiPM (a) and to the RGB-SiPM (b) arrays are presented. RGB-SiPM bias voltage was set in $124 \mathrm{~V}$ in order to compare the results at same overvoltage conditions. Better results were obtained with the NUV-SiPM array, and taking into account that the covered area is the same, and that the PDE in the emission range of the EJ-276G is very similar for both types of SiPM, the difference can be ascribed to the dark count rate of each type of SiPM (at OV $=4 \mathrm{~V}$ ), which is much lower in the case of NUV-SiPM (see Table 1). The assembly EJ$276 \mathrm{G} / \mathrm{NUV}$-SiPM shows a FoM value $\sim 75 \%$ respect to the one we obtained with the standard PMT, which is consistent with the small EJ-229 and EJ-276 results because the PDE is larger for those scintillators, so better performance is expected.
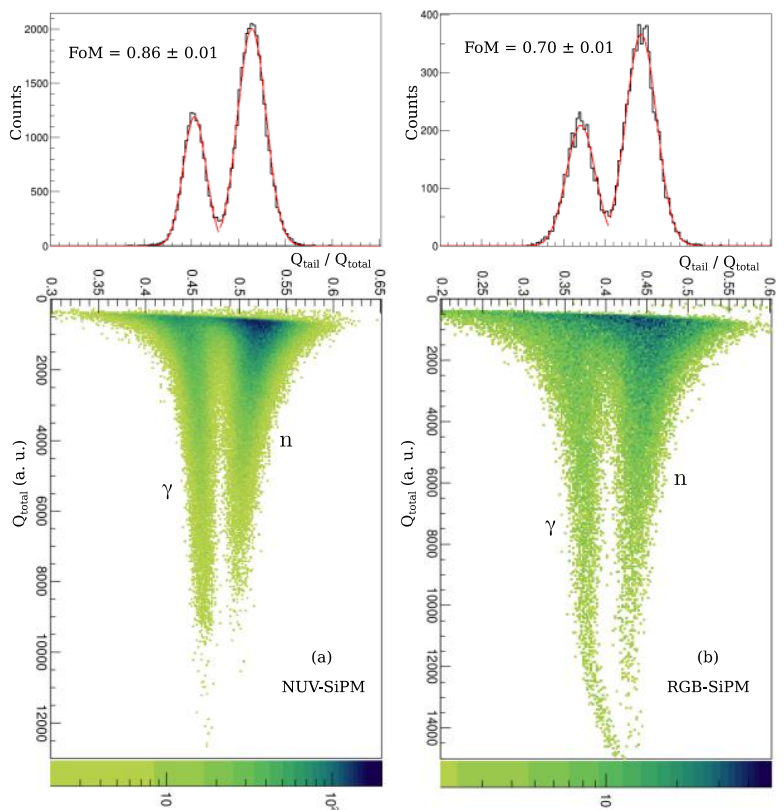

Fig. 8. 2D plots, PSP versus $Q_{\text {total }}$, corresponding to the measurements using the Am-Be source with the EJ-276G (25 mm dia. x $25 \mathrm{~mm}$ thick.) coupled to the NUV-SiPM (a) and to the RGB-SiPM array (b). PSP distributions and FoM values (at $\sim 1 \mathrm{MeVee}$ ) are reported on top.

\section{EJ-299, $50 \mathrm{~mm}$ dia. $x 50 \mathrm{~mm}$ thick.}

Finally, an incipient study of the $\mathrm{n} / \gamma$ discrimination using a large-sized plastic scintillator coupled to a SiPM is shown in this section. A 2" right cylinder EJ-299 scintillator was coupled to a NUV-SiPM array, i.e. covering only $\sim 15 \%$ of the scintillator face. The results are reported in Fig. 9. In this case, when the large scintillator is read-out with the NUV-SiPM the $\mathrm{n} / \gamma$ separation is poor (FoM $\sim 0.43$ at $\sim 1 \mathrm{MeVee}$ ), meaning that despite the high PDE exhibited by the SiPM, the light collection is very little to be able to get accurate signals. Following this idea we tried to use a conical light guide $(\mathrm{D} 1=50 \mathrm{~mm}, \mathrm{D} 2=20$ $\mathrm{mm}, \mathrm{H}=55 \mathrm{~mm}$ ) to improve the light collection.
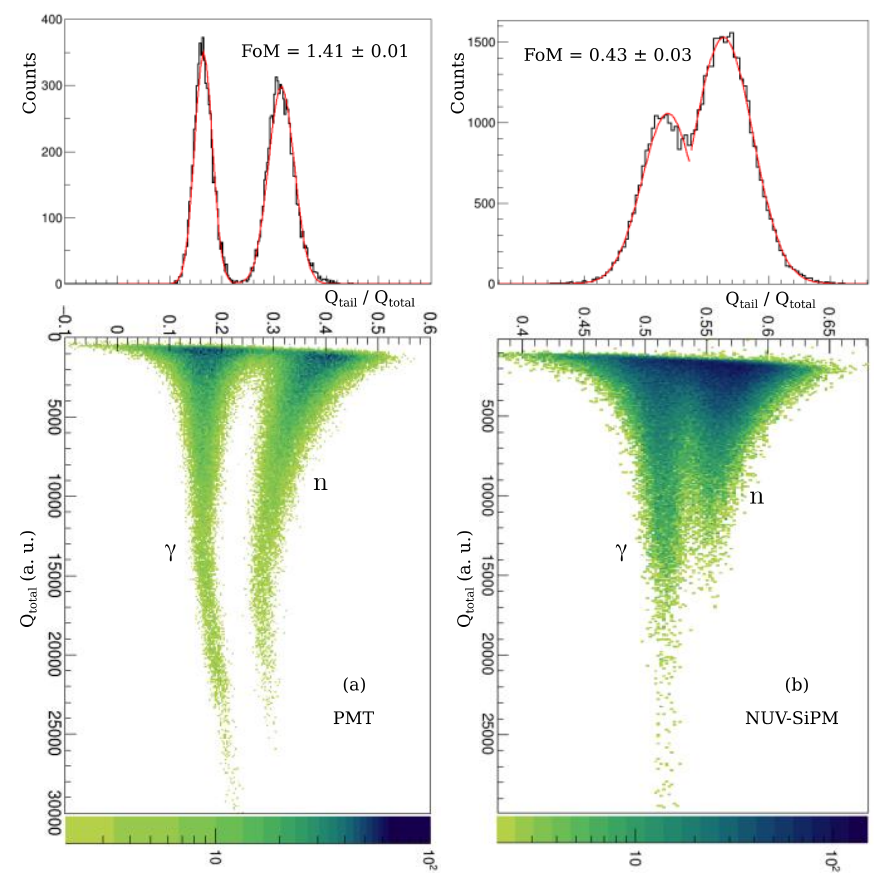

Fig. 9. 2D plots, PSP versus $Q_{\text {total }}$, corresponding to the measurements using the Am-Be source with the EJ-299 (50 mm dia. X $50 \mathrm{~mm}$ thick.) coupled to the PMT (a) and to the NUV-SiPM array (b). PSP distributions and FoM values (at $\sim 1 \mathrm{MeVee}$ ) are reported on top.

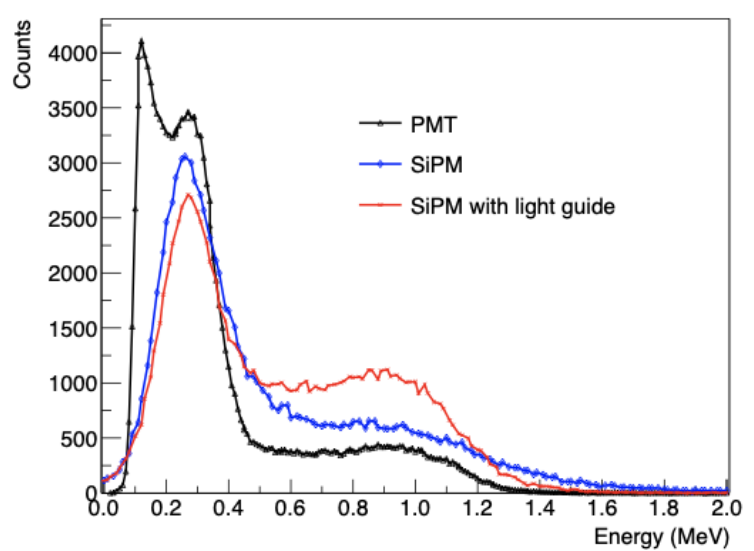

Fig. 10. ${ }^{22} \mathrm{Na}$ energy spectra corresponding to the 2" right cylinder of EJ-299 coupled to the PMT (H1949-51)and to the NUV-SiPM with and without light guide.

In Fig. 10 we show ${ }^{22} \mathrm{Na}$ spectra acquired with the large scintillator coupled to the PMT (black), to the NUV-SiPM without light guide (blue) and to the NUV-SiPM with light guide (red). Better energy resolution (at $\sim 1 \mathrm{MeV}$ ) was found using the light guide, however, neither with nor without light guide is possible to recognize the Compton edge of the $511 \mathrm{keV}$ photon (i.e. at $\sim 300 \mathrm{keV}$ ), meaning that the light collection is still too little. Furthermore, same FoM values were obtained with and without the light guide. These results suggest that in order to improve the $n / \gamma$ discrimination capability of the assembly large-sized plastic scintillator/SiPM array, the 
covered area should be increased. For example, four NUVSiPM array would cover $60 \%$ of the scintillator face, so, we could expect a performance similar to the 1" right cylinder of EJ-276 coupled to one NUV-SiPM array.

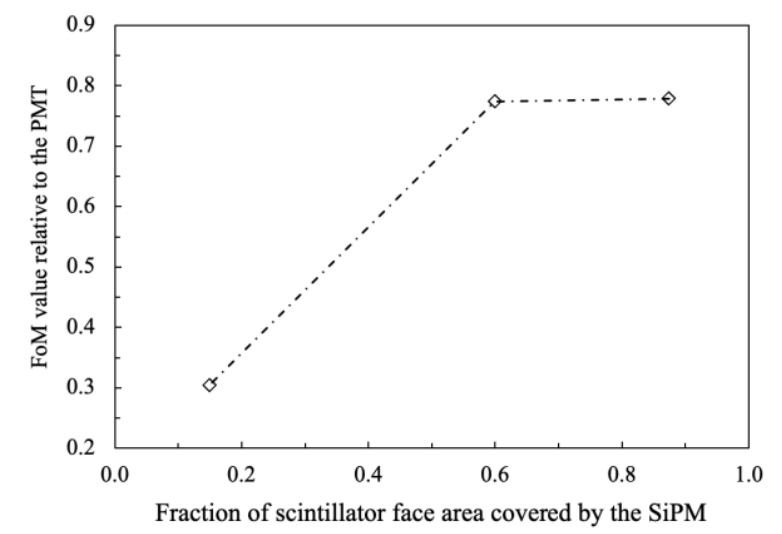

Fig. 11. Relationship between the performance of the detector assembly, in terms of $n / \gamma$ discrimination, as a function of the fraction of the scintillator face area covered by the SiPM.

\section{CONCLUSIONS}

The main purpose of this work was to develop a neutron/gamma detector prototype, based on a plastic scintillator read-out with a SiPM array, to be used as a secondary detector of the Active Target for SPES (at Legnaro National Laboratories, Italy). In order to achieve that, we have presented an innovative preamplifier designed to manage the sum of the individual signals and the bias supply of each pixel of $4 \times 4$ SiPM arrays, produced by AdvanSiD (Trento, Italy). We have studied the response of small, medium and large plastic scintillators with neutron/gamma discrimination capabilities coupled to a $4 \times 4$ SiPM array and to a standard 2" PMT. The small (EJ-299) and medium (EJ-276 and EJ-276G) plastic scintillators exhibit excellent $\mathrm{n} / \gamma$ discrimination (FoM values > 1) reaching almost $80 \%$ of the performance of a standard PMT. Besides, good light collection combined with the high PDE of the SiPM produced excellent results in terms of energy resolution. Furthermore, we have found that even if the EJ$267 \mathrm{G}$ scintillator is known as the green emitting EJ-276, its performance is better when it is coupled to the NUV-SiPM (because it has lower dark count rate). By the other hand, we have obtained poor $\mathrm{n} / \gamma$ discrimination with the large-sized EJ299 scintillator (2" right cylinder) when it is coupled to the NUV-SiPM. The main reason is the lack of light collection. As a summary, the relationship between the performance of the detector assembly, in terms of $n / \gamma$ discrimination, as a function of the fraction of the scintillator face area covered by the SiPM is presented in Fig. 11. It is clear that small scintillators are not useful for most of applications, and for that reason, the next steps will be adding SiPM arrays in order to increase the light collection and at the same time the discrimination performance (at least 3 or 4 arrays to cover more than $50 \%$ of the scintillator face). The other solution could be the use of brightest neutron detectors such as stilbene crystals or liquid scintillators.

\section{REFERENCES}

[1] G. Prete, A. Andrighetto, J. Esposito, P. Mastinu, J. Wyss, "The SPES project: a second generation ISOL facility," Physics Procedia, vol 26, pp. 274-283, 2012, 10.1016/j.phpro.2012.03.035.

[2] T. Roger, J. Pancin, G.F. Grinyer, B. Mauss, A.T. Laffoley, P. Rosier, et al., "Demonstrator Detection System for the Active Target and Time Projection Chamber (ACTAR TPC) Project," Nuclear Inst. and Methods in Physics Research, A, vol 895, pp. 126-134, 2018, 10.1016/j.nima.2018.04.003.

[3] B. Mauss, P. Morfouace, T. Roger, J. Pancin, G.F. Grinyer, J. Giovinazzo, et al., "Commissioning of the ACtive TARget and Time Projection Chamber (ACTAR TPC)," Nuclear Inst. and Methods in Physics Research, A, vol 940, pp. 498-504, 2019, 10.1016/j.nima.2019.06.067.

[4] M. Cinausero, V. L. Kravchuk, T. Marchi, F. Gramegna, G. Collazuol, G. de Angelis, et al., "Angular Distribution and Cross Section Measurement of the ${ }^{6} \mathrm{Li}\left({ }^{3} \mathrm{He}, \mathrm{n}\right){ }^{8} \mathrm{~B}$ Reaction at $5.8 \mathrm{MeV}$ ", EPJ Web of Conferences 66, 03048, 2014, 10.1051/epjconf/20146603048.

[5] J. J. Valiente-Dobón, A. Poves, A. Gadea, B. Fernández-Domínguez, "Broken mirror symmetry in ${ }^{36} \mathrm{~S}$ and ${ }^{36} \mathrm{Ca}$ ", Physical Review C, vol. 98, 011302(R), 2018, 10.1103/PhysRevC.98.011302.

[6] T. Cervi, M. Babicz, M. Bonesini, A. Falcone, A. Menegolli, G. L. Raselli, et al., "Characterization of SiPM arrays in different series and parallel configurations," Nuclear Inst. and Methods in Physics Research, A, vol 912, pp. 209-212, 2018, 10.1016/j.nima.2017.11.038.

[7] F. Pino, L. Stevanato, D. Cester, G. Nebbia, L. Sajo-Bohus, G. Viesti, "Detecting fast and thermal neutrons with a boron loaded liquid scintillator, EJ-339A," Applied Radiation and Isotopes, vol 92 pp. 6-11, 2014, 10.1016/j.apradiso.2014.05.025.

[8] C. L. Fontana, et al., "A Distributed Data Acquisition System for Signal Digitizers with On-Line Analysis Capabilities," in Proc. 2017 IEEE NSS/MIC, Atlanta, USA. 2017, pp. 1-5. 10.1109/NSSMIC.2017.8533063

[9] C. L. Fontana, et al., "Resource sharing in nuclear physics laboratory classes: A distributed data acquisition system for experiments with shared resources and data management," in Proc. 2017 CAARI, Texas, USA, 2019. p. 050024. 10.1063/1.5127716.

[10] C. L. Fontana, et al., “ A Distributed Data Acquisition System for Nuclear Detectors," in Proc. CRETE17, Crete, Greece. 2018. 10.1142/S2010194518601187.

[11] Can Liao, Haori Yang, "Pulse shape discrimination using EJ-299-33 plastic scintillator coupled with a Silicon Photomultiplier array," Nuclear Inst. and Methods in Physics Research, A, vol 789, pp. 150-157, 2015, 10.1016/j.nima.2015.04.016.

[12] M. P. Taggart, P. J. Sellin, "Comparison of the pulse shape discrimination performance of plastic scintillators coupled to a SiPM," Nuclear Inst. and Methods in Physics Research, A, vol 908, pp. 148-154, 2018, 10.1016/j.nima.2018.08.054.

[13] M. Grodzicka-Kobylka, T. Szczesniak, M. Moszyński, L. Swiderski, D. Wolski, J. Baszak, et al., "Study of $n-\gamma$ discrimination by zero-crossing method with SiPM based scintillation detectors," Nuclear Inst. and Methods in Physics Research, A, vol 883, pp. 159-165, 2018, 10.1016/j.nima.2017.11.045.

[14] F. Ferrulli, N. Dinar, L. G. Manzano, M. Labalme, M. Silari, "Characterization of stilbene and EJ-276 scintillators coupled with a large area SiPM array for a fast neutron dose rate detector," Nuclear Inst. and Methods in Physics Research, A, vol 1010, 165566, 2021, 10.1016/j.nima.2021.165566. 